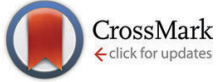

Cite this: Chem. Commun., 2015, 51,4290

Received 27th December 2014, Accepted 3rd February 2015

DOI: $10.1039 / c 4 c c 10357 b$

www.rsc.org/chemcomm

\section{A regioregular terpolymer comprising two electron-deficient and one electron-rich unit for ultra small band gap solar cells $\dagger$}

\author{
Weiwei Li, ${ }^{\star a b}$ Koen H. Hendriks, ${ }^{\text {bc }}$ Alice Furlan, ${ }^{\text {bc }}$ Andong Zhang, ${ }^{a}$ \\ Martijn M. Wienk ${ }^{\mathrm{bc}}$ and René A. J. Janssen ${ }^{\mathrm{bc}}$
}

\begin{abstract}
A regioregular terpolymer that combines electron-deficient diketopyrrolopyrrole and benzothiadiazole units with an electron-rich dithienopyrrole unit is presented. In solar cells, the terpolymer affords an open circuit voltage of $0.52 \mathrm{~V}$ and a power conversion efficiency of $3.7 \%$ with a spectral response up to $1050 \mathrm{~nm}$.
\end{abstract}

Bulk heterojunction polymer solar cells (PSCs) with conjugated polymers as electron donor and fullerene derivatives such as $[6,6]$-phenyl- $\mathrm{C}_{71}$-butyric acid methyl ester ([70]PCBM) as electron acceptor have significantly progressed in the last decade and now reach power conversion efficiencies (PCEs) above $10 \%$ in single junction solar cells. ${ }^{1-3}$ The efficiencies have been further increased to $11-12 \%$ via tandem and triple junction device architectures, ${ }^{4,5}$ in which two or three photoactive layers with complementary absorption spectra are stacked and connected in series. By applying different conjugated polymers that absorb light from the visible light to the near-infrared region above $1000 \mathrm{~nm}$, the conversion efficiencies of triple junction solar cells may achieve $15-20 \%{ }^{6}$ To reach this ambitious goal, it will be important to develop efficient conjugated polymers that absorb up to $1000 \mathrm{~nm}$ and beyond.

Conjugated polymers with tailored optical band gaps $\left(E_{\mathrm{g}}\right)$ can be obtained using electron donating and electron deficient units alternating along the main chain. In general stronger electron donors and stronger acceptors reduce the optical band gap. Using this design motif and by employing suitable side chains, the energy levels, solubility, aggregation and crystallinity of the polymers can be tuned. Alternating conjugated polymers

\footnotetext{
${ }^{a}$ Beijing National Laboratory for Molecular Sciences, CAS Key Laboratory of Organic Solids, Institute of Chemistry, Chinese Academy of Sciences, Beijing 100190, P. R. China.E-mail: liweiwei@iccas.ac.cn

${ }^{b}$ Molecular Materials and Nanosystems, Institute of Complex Molecular Systems, Eindhoven University of Technology, P. O. Box 513, 5600 MB Eindhoven, The Netherlands. E-mail: r.a.j.janssen@tue.nl

${ }^{c}$ Dutch Institute for Fundamental Energy Research, De Zaale 20, 5612 AJ Eindhoven, The Netherlands

$\dagger$ Electronic supplementary information (ESI) available. See DOI: 10.1039/ c4cc10357b
}

have already reached high performance in polymer solar cells, especially for wide $\left(E_{\mathrm{g}}>1.7 \mathrm{eV}\right)$ and medium $\left(1.7 \mathrm{eV}>E_{\mathrm{g}}>\right.$ $1.4 \mathrm{eV}$ ) optical band gaps with PCEs above $9 \% .^{1-3} \mathrm{~A}$ few small band gap conjugated polymers with $E_{\mathrm{g}}$ close to $1.4 \mathrm{eV}$ provide $\geq 8 \%$ PCE. $^{7-9}$ For $E_{\mathrm{g}}$ below $1.25 \mathrm{eV}$ the most efficient cells reach $5.3 \%$ PCE. $^{10}$ In many of the successful materials a significant photon energy loss between $E_{\mathrm{g}}$ and open circuit voltage $\left(V_{\mathrm{oc}}\right)$ is incurred, $E_{\text {loss }}=E_{\mathrm{g}}-q V_{\mathrm{oc}}$. $E_{\text {loss }}$ provides a driving force for photoinduced charge generation and often enhances the short circuit current density $\left(J_{\mathrm{sc}}\right)$. When the optical band gap is reduced, charge generation is usually negligible when $E_{\text {loss }}$ becomes less than $0.6 \mathrm{eV} .{ }^{11}$ This problem becomes especially important when the absorption of conjugated polymers extends above $1000 \mathrm{~nm}$ where the optical band gap becomes less than $1.2 \mathrm{eV}$. To maintain a high quantum efficiency for charge generation, the $V_{\mathrm{oc}}$, which is controlled by the energy difference between the HOMO of the donor and the LUMO of the acceptor, must be designed to be less than $0.6 \mathrm{~V}$. However, a too low $V_{\mathrm{oc}}$ would quickly lead to an insignificant PCE. Hence, conjugated polymers with a very small band gap require a judicious design in terms of energy levels to be applicable in PSCs.

Several conjugated polymers with absorption beyond $1000 \mathrm{~nm}$ have been reported, often using strong electron deficient units such as thienoisoindigo, ${ }^{12,13}$ thiadiazoloquinoxaline, ${ }^{14,15}$ bisbenzo thiadiazole, ${ }^{16}$ pyrazinoquinoxaline ${ }^{17,18}$ or diketopyrrolopyrrole (DPP). ${ }^{19}$ When applied in polymer solar cells PCEs up to 5.3\% have been obtained. ${ }^{10}$ Especially, PDPP2T-DTP (Fig. 1a) with DPP as electron acceptor and dithienopyrrole (DTP) as strong electron donor affords $E_{\mathrm{g}}=1.23 \mathrm{eV}^{19}$ and provides $J_{\mathrm{sc}}>20 \mathrm{~mA} \mathrm{~cm}{ }^{-2} \cdot{ }^{10}$ The $V_{\mathrm{oc}}$ of cells based on this polymer is, however, relatively low with $0.43 \mathrm{~V}$, resulting in $E_{\text {loss }}=0.80 \mathrm{eV}$. In order to reduce $E_{\text {loss }}$ and enhance $V_{\mathrm{oc}}$, we introduce the use of two electron deficient units, DPP and benzothiadiazole (BT), in combination with DTP as electron donor. The new ultra low band gap polymer, PDPP2T-BT-co-DTP (Fig. 1c) provides a significantly higher $V_{\mathrm{oc}}$ of $0.52 \mathrm{eV}$ and PCE of $3.7 \%$ with a reduced optical band gap of $1.19 \mathrm{eV}$ in polymer solar cells.

PDPP2T-DTP ${ }^{10}$ and PDPP2T-BT ${ }^{20}$ were synthesized following previously reported procedures. The regioregular alternating 

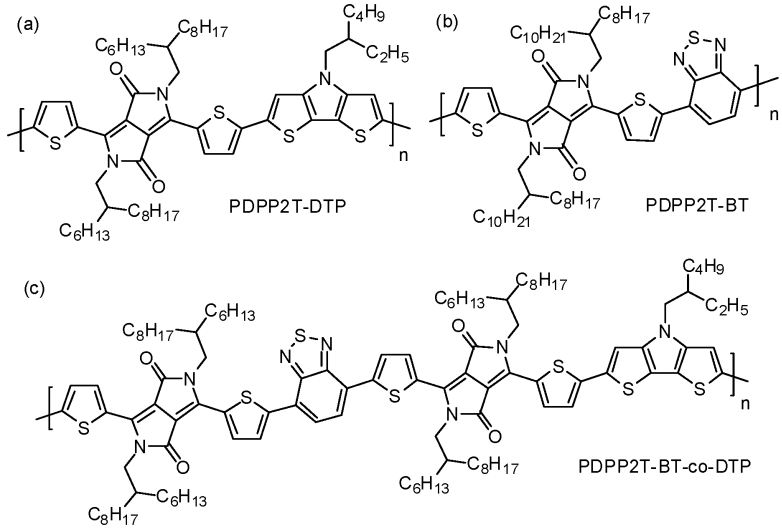

Fig. 1 The DPP polymers with (a) dithienopyrrole (DTP), (b) benzothiadiazole (BT) and (c) both DTP and BT.

copolymer PDPP2T-BT-co-DTP was synthesized using the dibromo-monomer of T-DPP-T-BT-T-DPP-T and the bisstannylDTP monomer via Stille polymerization (Scheme S1, ESI $\dagger$ ). The conditions applied in the polymerization reaction, toluene/DMF $10: 1(\mathrm{v} / \mathrm{v})$ as solvent and $\mathrm{Pd}_{2}(\mathrm{dba})_{3} / \mathrm{PPh}_{3} 1: 4(\mathrm{~mol} / \mathrm{mol})$ as catalyst, were chosen to reduce homo coupling defects and achieve a high molecular weight. ${ }^{21}$ The viscous gel formed during polymerization indicates a high molecular weight and the work-up required the use of 1,1,2,2-tetrachloroethane at $140{ }^{\circ} \mathrm{C}$. It was not possible to measure the molecular weight by gel permeation chromatography (GPC) in $o$-dichlorobenzene ( $o$-DCB) because the polymer seems to adhere to or decompose on the column. The same problem was previously encountered for PDPP2T-DTP. ${ }^{10}$ The thermal stability of PDPP2T-BT-co-DTP is somewhat less than that of PDPP2T-BT and PDPP2T-DTP (Fig. S1, ESI $\dagger$ ). The strong electron donating DTP unit makes polymers based on DTP susceptible to oxidation in air, and processing of devices was performed under inter atmosphere. ${ }^{10}$

All three polymers absorb in the near-infrared region with onsets above $1000 \mathrm{~nm}$ (Fig. 2a). The absorption in thin films is slightly red-shifted compared to chloroform solution (Fig. S2, ESI $\dagger$ ). PDPP2T-BT has a lower band gap (1.19 eV) than PDPP2TDTP $\left(E_{\mathrm{g}}=1.23 \mathrm{eV}\right)$. While we anticipated that the combination of two acceptors (DPP and BT) would increase the electron withdrawing character and could further reduce the band gap, the terpolymer PDPP2T-BT-co-DTP, that combines DPP, BT, and DTP also has $E_{\mathrm{g}}=1.19 \mathrm{eV}$.

Importantly, however, the introduction of two acceptors allows to effectively tune the frontier orbital energy levels. ${ }^{22,23}$
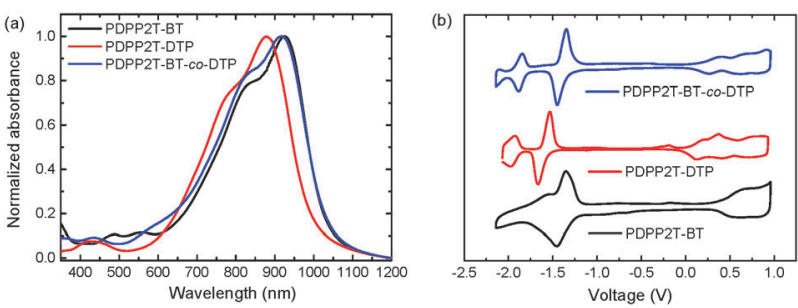

Fig. 2 (a) Optical absorption spectra of the polymers in solid state films and (b) cyclic voltammograms of the polymers thin films on ITO substrates.
Table 1 Optical and electrochemical properties of the polymers

\begin{tabular}{lllllll}
\hline Polymer & $\begin{array}{l}E_{\mathrm{g}}^{\mathrm{CHCl}_{3}} \\
(\mathrm{eV})\end{array}$ & $\begin{array}{l}E_{\mathrm{g}}^{\mathrm{fim}} \\
(\mathrm{eV})\end{array}$ & $\begin{array}{l}E_{\mathrm{LUMO}}{ }^{a} \\
(\mathrm{eV})\end{array}$ & $\begin{array}{l}E_{\mathrm{HOMO}}{ }^{a} \\
(\mathrm{eV})\end{array}$ & $\begin{array}{l}E_{\mathrm{g}}^{\mathrm{cv}} \\
(\mathrm{eV})\end{array}$ & $\begin{array}{l}\Delta E_{\mathrm{LL}}{ }^{b} \\
(\mathrm{eV})\end{array}$ \\
\hline PDPP2T-BT & 1.22 & 1.19 & -4.01 & -5.55 & 1.54 & 0.15 \\
PDPP2T-DTP & 1.26 & 1.23 & -3.68 & -5.26 & 1.58 & 0.48 \\
PDPP2T-BT-co-DTP & 1.21 & 1.19 & -3.92 & -5.35 & 1.43 & 0.24
\end{tabular}

${ }^{a}$ Determined using a work function value of $-5.23 \mathrm{eV}$ for Fc/Fc ${ }^{+} .{ }^{b} \Delta E_{\mathrm{LL}}=$ $q\left(E_{\text {LUMO }}([70] \mathrm{PCBM})-E_{\text {LUMO }}\right)$ with $E_{\text {LUMO }}([70] \mathrm{PCBM})=-4.16 \mathrm{~V}$.

Cyclic voltammetry (Fig. $2 \mathrm{~b}$ and Table 1 ) reveals that as a consequence of incorporating two electron deficient units (DPP and BT), the PDPP2T-BT copolymer has deep HOMO and LUMO levels $(-5.55 \mathrm{eV}$ and $-4.01 \mathrm{eV})$. Hence, the LUMO-LUMO offset ( $\left.\Delta E_{\mathrm{LL}}\right)$ between PDPP2T-BT and [70]PCBM is only $0.15 \mathrm{eV}$ and significantly less than the $\sim 0.3 \mathrm{eV}$ threshold for charge separation. This problem often occurs in very small band gap conjugated polymers. ${ }^{24,25}$ With one electron deficient (DPP) and one electron rich unit (DTP), the PDPP2T-DTP copolymer exhibits significantly less negative HOMO and LUMO levels $(-5.26 \mathrm{eV}$ and $-3.68 \mathrm{eV})$ to give $\Delta E_{\mathrm{LL}}=0.48 \mathrm{eV}$. The new terpolymer PDPP2T-BT-co-DTP has the HOMO and LUMO in between those of the two copolymers PDPP2T-BT and PDPP2T-DTP, so that $\Delta E_{\mathrm{LL}}=0.24 \mathrm{eV}$. The low lying HOMO level will help to achieve a higher $V_{\text {oc }}$ than that of PDPP2T-DTP, while the higher $\Delta E_{\mathrm{LL}}$ than that of PDPP2T-BT is beneficial for the efficiency of photoinduced charge separation.

The three polymers were applied in polymer solar cells with a transparent ITO/poly(3,4-ethylenedioxy thiophene):poly(styrene sulfonate) (PEDOT:PSS) bottom contact for hole collection and $\mathrm{LiF} / \mathrm{Al}$ top contact for electron collection. The photoactive layers, in which the polymers were blended with [70]PCBM, were carefully optimized for the donor to acceptor weight ratio, solvent, co-solvent, and thickness. In general, the optimized donoracceptor ratio is $1: 2(\mathrm{w} / \mathrm{w})$ and the optimal thickness was around $110 \mathrm{~nm}$, when spin coating from chloroform solution. For PDPP2T-BT 10\% $o$-DCB as co-solvent gave the best performance. For PDPP2T-DTP and PDPP2T-BT-co-DTP the best co-solvents concentrations are $5 \% \quad o$-DCB and $3 \%$ 1-chloronaphthalene (1-CN), respectively. The $J-V$ characteristics and external quantum efficiencies (EQEs) of the optimized devices are depicted in Fig. 3 and the device parameters are summarized in Table 2.

As expected from the low $\Delta E_{\mathrm{LL}}$ the PDPP2T-BT copolymer gives a low PCE of $1.2 \%$, mainly due to a low short circuit
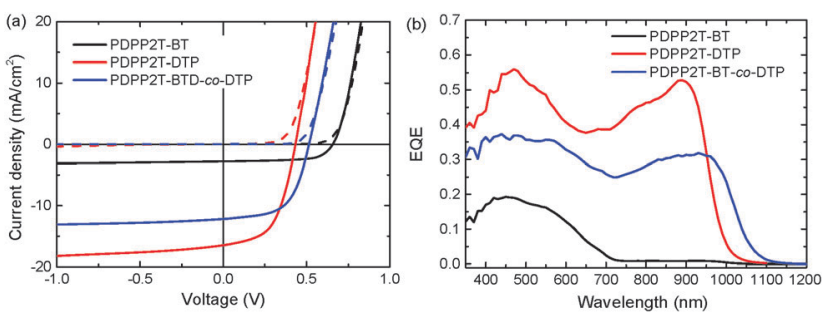

Fig. 3 (a) $J-V$ characteristics in dark (dashed lines) and under white light illumination (solid lines) of optimized solar cells of the polymers with [70]PCBM. (b) EQE of the same devices. 
Table 2 Solar cell parameters of optimized solar cells of the DPP polymers with [70]PCBM

\begin{tabular}{lcllll}
\hline Polymer & $J_{\mathrm{sc}}{ }^{a}\left(\mathrm{~mA} \mathrm{~cm}^{-2}\right)$ & $V_{\mathrm{oc}}(\mathrm{V})$ & $\mathrm{FF}$ & PCE $^{a}(\%)$ & $E_{\text {loss }}(\mathrm{eV})$ \\
\hline PDPP2T-BT & 2.8 & 0.66 & 0.66 & 1.2 & 0.53 \\
PDPP2T-DTP & 16.6 & 0.43 & 0.54 & 3.9 & 0.80 \\
PDPP2T-BT-co-DTP & 12.2 & 0.52 & 0.58 & 3.7 & 0.67
\end{tabular}

${ }^{a} J_{\text {sc }}$ and PCE were calculated by integrating the EQE spectrum with the AM1.5G spectrum.

current density $\left(J_{\mathrm{sc}}=2.8 \mathrm{~mA} \mathrm{~cm}{ }^{-2}\right)$. The cells have a high $V_{\mathrm{oc}}$ of $0.66 \mathrm{~V}$ at this low band gap $(1.19 \mathrm{eV})$. The photon energy loss $E_{\text {loss }}$ equals $0.53 \mathrm{eV}$ and is below the threshold of $0.6 \mathrm{eV}$ for efficient charge transfer, ${ }^{11}$ rationalizing the low $J_{\mathrm{sc}}$. In contrast, photovoltaic devices based on the copolymer PDPP2T-DTP give a high $J_{\mathrm{sc}}$ of $16.6 \mathrm{~mA} \mathrm{~cm}^{-2}$ and PCE of $3.9 \%$, but a relatively low $V_{\mathrm{oc}}$ of $0.43 \mathrm{~V}$ as consequence of the higher lying HOMO. The low $V_{\mathrm{oc}}$ increases the photon energy loss to $E_{\text {loss }}=0.80 \mathrm{eV}$. The new terpolymer PDPP2T-BT-co-DTP gives $V_{\mathrm{oc}}=0.52 \mathrm{~V}$, i.e. in between the $V_{\mathrm{oc}}$ 's obtained with the two copolymers, but a somewhat low $J_{\text {sc }}\left(12.2 \mathrm{~mA} \mathrm{~cm}^{-2}\right)$ and PCE of $3.7 \%$. Here $E_{\text {loss }}$ is $0.67 \mathrm{eV}$. The differences in photocurrent are also reflected in the EQE spectra (Fig. 3b). In PDPP2T-BT:[70]PCBM cells the contribution of light absorbed by the polymer in the 700-1050 nm region is negligible and photocurrent is only created via excitation of [70]PCBM. In contrast, a broad spectral response from 350 to $1050 \mathrm{~nm}$, covering the absorption spectra of fullerene and polymer is found for PDPP2T-BT-co-DTP:[70]PCBM blends. Unfortunately, the PDPP2T-BT-co-DTP terpolymer provides a relatively low EQE (maximizing at 0.32 ) compared to that of the PDPP2T-DTP copolymer (maximizing at 0.53).

As previously reported, a lower energy loss decreases the driving force for electron transfer and correlates with a lower EQE. ${ }^{7}$ Hence, the lower EQE of the terpolymer PDPP2T-BT-coDTP is possibly related to the reduced $\Delta E_{\mathrm{LL}}$ and $E_{\text {loss. }}$. However, the energy loss of the terpolymer is $0.67 \mathrm{eV}$, i.e. above the threshold of $0.6 \mathrm{eV} \cdot{ }^{11}$ Actually, another DPP-polymer, PDPP3T, where $E_{\text {loss }}=0.65 \mathrm{eV}$ gave a maximum EQE of $0.50,{ }^{7}$ which is significantly higher than the 0.32 of the terpolymer. Therefore other effects than $E_{\text {loss }}$ likely contribute to the low EQE.

First, TEM reveals that the morphology of the PDPP2T-BT- $c$ DTP:[70]PCBM blends consists of semicrystalline polymer
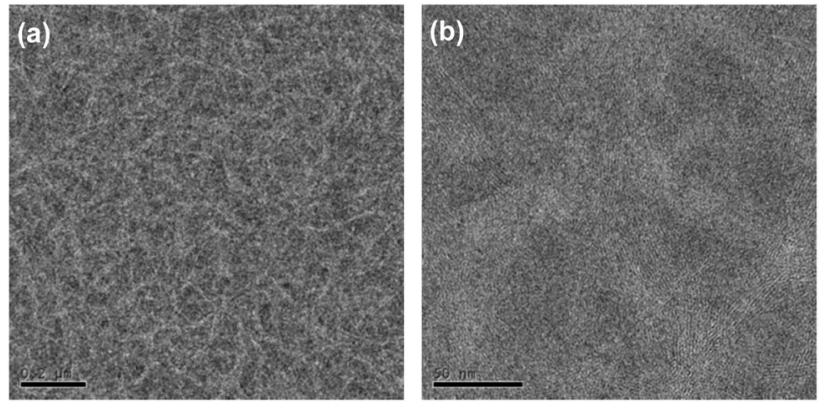

Fig. 4 Bright field TEM images of the optimized PDPP2T-BTD-co-DTP: [70]PCBM blend films. Scale bars: (a) $200 \mathrm{~nm}$ and (b) $50 \mathrm{~nm}$. fibrils (Fig. 4). The crystallinty can be readily seen from the lattice fringes in Fig. $4 \mathrm{~b}$. The width of these semicrystalline fibrils exceeds $10 \mathrm{~nm}$, commonly considered as the maximum exciton diffusion length. We have shown recently for a range of DPP-polymers that the fibril width anti-correlates with the EQE and that exciton loss via intrinsic decay may contribute to a low EQE. ${ }^{26}$

Second, localization of excitons on the T-DPP-T-BT-T-DPP-T segments may occur. Because PDPP2T-BT has a smaller band gap than PDPP2T-DTP, excitons may prefer T-DPP-T-BT-TDPP-T segments. Excitons on such segments may have a lower tendency to transfer an electron to the fullerene, as suggested by the fact that the PDPP2T-BT copolymer is unable to create charges efficiently. This explanation is supported by our recent work on the detrimental effect of low-LUMO chain segments on the charge generation efficiency. ${ }^{21}$

In conclusion, a new conjugated terpolymer with an ultra small band gap, $E_{\mathrm{g}}=1.19 \mathrm{eV}$, was designed and applied in polymer solar cells. By incorporating two electron-deficient and one electron-rich unit into the conjugated backbone the frontier energy levels were tailored to maximize $V_{\mathrm{oc}}$ and $J_{\mathrm{sc}}$. The terpolymer provided a relatively high $V_{\mathrm{oc}}(0.52 \mathrm{~V})$ and PCE $(3.7 \%)$ with a photoresponse up to $1050 \mathrm{~nm}$, despite a moderate EQE. The study shows that by extending the push-pull approach and incorporating more units than single electron rich and electron poor units is viable way to create semiconducting polymers with finely tuned energy levels.

The work was performed in the framework of the Large cells and X10D projects that received funding from the European Commission's Seventh Framework Programme (Grant Agreement No. 261936 and No. 287818). The research forms part of the Solliance OPV programme and has received funding from the Ministry of Education, Culture and Science (Gravity program 024.001.035).

\section{Notes and references}

1 Y. Liu, J. Zhao, Z. Li, C. Mu, W. Ma, H. Hu, K. Jiang, H. Lin, H. Ade and H. Yan, Nat. Commun., 2014, 5, 5293.

2 J.-D. Chen, C. Cui, Y.-Q. Li, L. Zhou, Q.-D. Ou, C. Li, Y. Li. and J.-X. Tang, Adv. Mater., 2015, 27, 1035-1041.

3 S.-H. Lia, H.-J. Jhuo, P.-N. Yeh, Y.-S. Cheng, Y.-L. Li, Y.-H. Lee, S. Sharma and S.-A. Chen, Sci. Rep., 2014, 4, 6813.

4 C.-C. Chen, W.-H. Chang, K. Yoshimura, K. Ohya, J. You, J. Gao, Z. Hong and Y. Yang, Adv. Mater., 2014, 26, 5670-5677.

5 A. R. bin Mohd Yusoff, D. Kim, H. P. Kim, F. K. Shneider, W. J. da Silva and J. Jang, Energy Environ. Sci., 2015, 8, 303-316.

6 R. A. J. Janssen and J. Nelson, Adv. Mater., 2013, 25, 1847-1858.

7 K. H. Hendriks, G. H. L. Heintges, V. S. Gevaerts, M. M. Wienk and R. A. J. Janssen, Angew. Chem., Int. Ed., 2013, 52, 8341-8344.

8 L. Dou, C.-C. Chen, K. Yoshimura, K. Ohya, W.-H. Chang, J. Gao, Y. Liu, E. Richard and Y. Yang, Macromolecules, 2013, 46, 3384-3390.

9 R. S. Ashraf, I. Meager, M. Nikolka, M. Kirkus, M. Planells, B. C. Schroeder, S. Holliday, M. Hurhangee, C. B. Nielsen, H. Sirringhaus and I. McCulloch, J. Am. Chem. Soc., 2015, 137, 1314-1321.

10 K. H. Hendriks, W. Li, M. M. Wienk and R. A. J. Janssen, J. Am. Chem. Soc., 2014, 136, 12130-12136.

11 D. Veldman, S. C. J. Meskers and R. A. J. Janssen, Adv. Funct. Mater., 2009, 19, 1939-1948.

12 G. W. P. Van Pruissen, F. Gholamrezaie, M. M. Wienk and R. A. J. Janssen, J. Mater. Chem., 2012, 22, 20387-20393.

13 M. Karakawa and Y. Aso, Macromol. Chem. Phys., 2013, 214, 2388-2397. 
14 A. P. Zoombelt, M. Fonrodona, M. M. Wienk, A. B. Sieval, J. C. Hummelen and R. A. J. Janssen, Org. Lett., 2009, 11, 903-906.

15 T. T. Steckler, P. Henriksson, S. Mollinger, A. Lundin, A. Salleo and M. R. Andersson, J. Am. Chem. Soc., 2013, 136, 1190-1193.

16 J. Fan, J. D. Yuen, M. J. Seifter, J.-H. Seo, A. R. Mohebbi, D. Zakhidov, A. Heeger and F. Wudl, Adv. Mater., 2012, 24, 2186-2190.

17 E. Wang, L. Hou, Z. Wang, S. Hellström, W. Mammo, F. Zhang, O. Inganäs and M. R. Andersson, Org. Lett., 2010, 12, 4470-4473.

18 T. L. D. Tam, T. Salim, H. Li, F. Zhou, S. G. Mhaisalkar, H. Su, Y. M. Lam and A. C. Grimsdale, J. Mater. Chem., 2012, 22, 18528-18534.

19 E. J. Zhou, J. Z. Cong, K. Hashimoto and K. Tajima, Energy Environ. Sci., 2012, 5, 9756-9759.

20 S. Cho, J. Lee, M. H. Tong, J. H. Seo and C. Yang, Adv. Funct. Mater., 2011, 21, 1910-1916.
21 K. H. Hendriks, W. Li, G. H. L. Heintges, G. W. P. van Pruissen, M. M. Wienk and R. A. J. Janssen, J. Am. Chem. Soc., 2014, 136, 11128-11133.

22 D. Dang, W. Chen, R. Yang, W. Zhu, W. Mammo and E. Wang, Chem. Commun., 2013, 49, 9335-9337.

23 W. Sun, Z. Ma, D. Dang, W. Zhu, M. R. Andersson, F. Zhang and E. Wang, J. Mater. Chem. A, 2013, 1, 11141-11144.

24 A. P. Zoombelt, S. G. J. Mathijssen, M. G. R. Turbiez, M. M. Wienk and R. A. J. Janssen, J. Mater. Chem., 2010, 20, 2240-2246.

25 F. L. Zhang, J. Bijleveld, E. Perzon, K. Tvingstedt, S. Barrau, O. Inganäs and M. R. Andersson, J. Mater. Chem., 2008, 18, 5468-5474.

26 W. Li, K. H. Hendriks, A. Furlan, W. S. C. Roelofs, M. M. Wienk and R. A. J. Janssen, J. Am. Chem. Soc., 2013, 135, 18942-18948. 